\title{
Can treatment delay be utilized as a key variable for monitoring the pool of infectious tuberculosis in a population?
}

\author{
Dag G. Storla ${ }^{1,2 *}$, Solomon Yimer ${ }^{1 *}$, and Gunnar A. Bjune ${ }^{1}$ \\ ${ }^{1}$ Department of International Health, Institute of General Practice and Community Medicine, University of Oslo, Norway \\ ${ }^{2}$ Centre for Imported and Tropical Diseases, Ullevål University Hospital, Oslo Norway \\ * Both authors contributed equally.
}

\begin{abstract}
Background: A major goal of tuberculosis control programs is to stop community transmission of Mycobacterium tuberculosis. However, this can not be rapidly accomplished because, in endemic areas, most of the population is already infected, serving as a reservoir that continuously contributes to the pool of infectious cases. Tuberculin surveys are the main tools used to monitor the infectious pool, but there are serious methodological constraints, and they require resources and expertise that are often unavailable. There is an urgent need for alternative means to monitor the epidemic at the local level.

Methodology: We investigated whether a systematic registration of treatment delay in the tuberculosis program records of the Amhara Region of Ethiopia could be utilized to estimate the infectious pool of tuberculosis.

Results: The study showed that the total number of infectious days and hence an estimate of the infectious pool could be calculated by recording the treatment delay for new TB cases, retreatment cases and failures, and by estimating the number of undiagnosed cases . Of these categories, treatment delay among new smear-positive tuberculosis cases contributes the greatest number of infectious days.

Conclusions: A local tuberculosis program can use a systematic recording of treatment delay as a quantifiable variable to monitor the infectious pool, and can also serve as a key indicator of program performance.
\end{abstract}

Key words: tuberculosis, diagnostic delay, treatment delay, Community Health Services, health surveys

J Infect Dev Ctries 2010; 4(2):083-090.

(Received 19 June 2009 - Accepted 21 September 2009)

Copyright (C) 2011 Storla et al. This is an open-access article distributed under the Creative Commons Attribution License, which permits unrestricted use, distribution, and reproduction in any medium, provided the original work is properly cited.

\section{Introduction}

The goal of tuberculosis (TB) control programs is to stop community transmission of Mycobacterium tuberculosis. However, this is a slow process, because more than $80 \%$ of the adult population in endemic areas is already infected [1,2] and serves as a reservoir that continuously contributes to the pool of infectious cases. The size of the infectious pool is fundamental to predict the TB epidemic.

The main indicator used by the World Health Organization (WHO) is the Case Detection Rate (CDR), which is defined as the notification rate of new cases of smear-positive TB divided by the estimated incidence rate. The WHO has defined a CDR of $70 \%$ as a target under the Millennium Development Goals [3].

Incidence rates and Annual Risk of Tuberculosis Infection (ARTI) are estimated based on tuberculin surveys utilizing Purified Protein Derivative (PPD) skin-tests, which have a range of methodological problems. The most serious is the low specificity, with a substantial cross-sensitization from BCG and environmental mycobacteria [4-7]. In an attempt to overcome these problems, the so-called models of mixture analysis have been developed to sort out the parameters of the underlying distributions [8]. But these models do not solve the problems.

The sensitivity of the tuberculin skin tests in surveys is substantially easier to define than their specificity. Close to normal distributions of reaction sizes have been found in healthy persons in areas with low interference from environmental mycobacteria [9]. Given a normal distribution, the number of infected individuals missed because they have an induration less than the cut-off value can be calculated. Regarding culture confirmed TB patients, however, there are diverging reports of sensitivity. A normal distribution is found [10,11], and using the national cut-off values, miscellaneous studies have found from $0-37 \%$ of culture-confirmed TB patients with a negative PPD skin test [12-14]. 
Figure 1. An illustration of the Dye model on tuberculosis case detection.

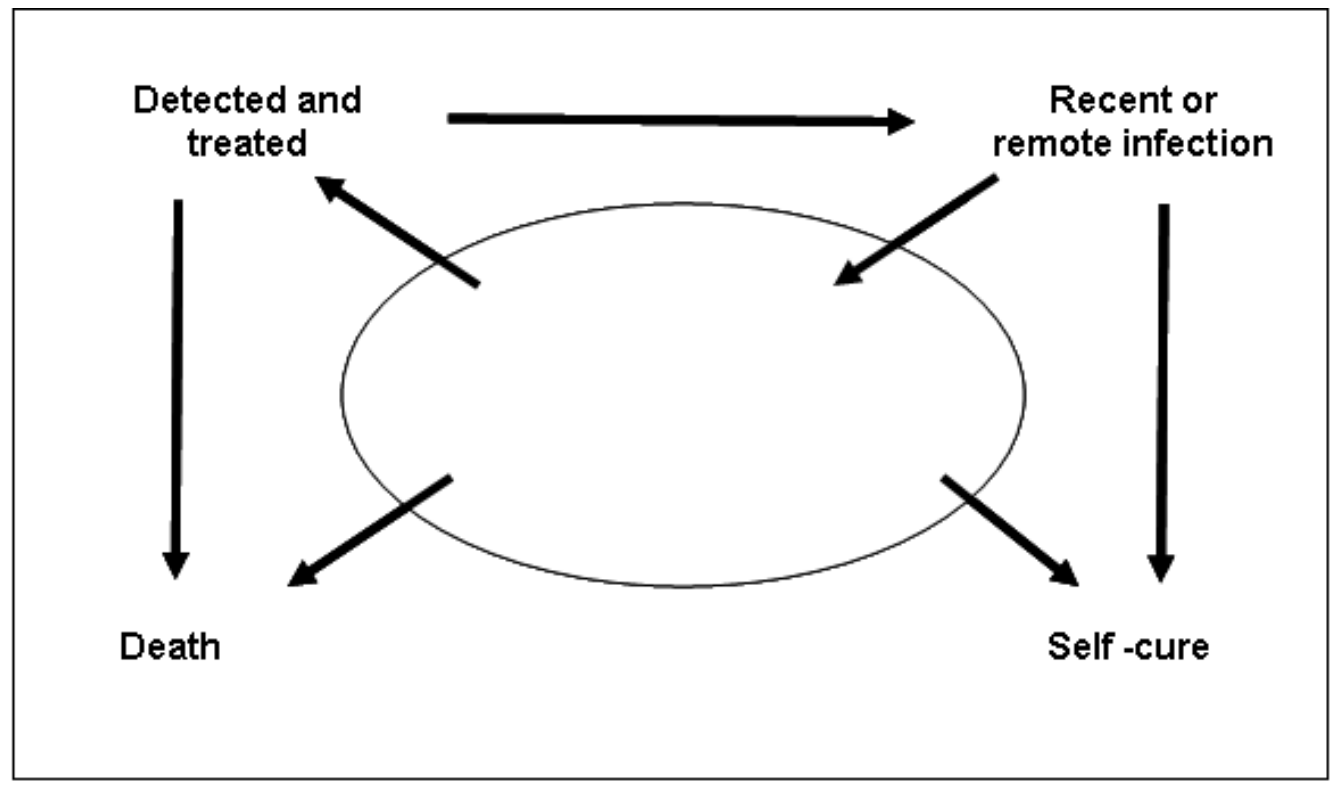

The arrows depict rates.

There is also a problem with inconsistent results depending on how the test is performed and the tuberculin reagent used. In Nigeria, 488 unvaccinated young workers with a high prevalence of tuberculosis were skin tested with 4 low-dose Mantoux tests (PPD-RT-23, PPD-Tuberculin, PPDBattey, and PPD-Gause) and with concentrated (2 $\mathrm{mg} / \mathrm{ml}$ ) PPD by the Heaf multiple-puncture method. Established methods for estimating the proportion of tuberculous-infected (cut-off point, dual-test, and curve-reconstruction) subjects yielded a wide range from 32\%-62\% found to be infected [15]. Diverging results depending on the tuberculin reagent have also been found in other studies [16-18]. Vaccination technique and BCG strain used by the national immunization program are also determinants of the PPD reaction [19].

Other studies report substantial inconsistent results over time. In 2000, Dubuis et al. performed a survey among 7,600 children aged 6-7

years in Kabul, Afghanistan. The average ARTI was estimated to be $0.34 \%$ [20]. The estimated ARTI from two national tuberculin surveys conducted in 1963 and 1978 were 2,55\% and 3,53\% respectively [21]. There are no major methodological arguments against the Dubuis survey. As seen elsewhere, one should expect the ARTI to rise in a country so heavily burdened by wars and hardship during the last decades [22]. There are no apparent reasons to explain the abrupt fall to $0.34 \%$ in 2000 , and these results should make us cautious in using skin test surveys as a tool of calculating disease burdens.

In the majority of low-income countries, tuberculin skin testing is not feasible. In addition to disposable needles and syringes, tuberculin itself is expensive. The costs of employing and training personnel on a national scale are tremendous. Additionally, the world also faces an increasing shortage of supplies of reference tuberculin [23].

Even finding an optimal method to determine the prevalence and risk of infection does not address the next main problem: What is the linkage between risk of infection and burden of disease? Utilizing a database of Dutch recruits of the pre-chemotherapy era, Styblo estimated that a $1 \%$ annual risk for infection would correspond with an incidence of new cases of smear-positive TB of approximately 50 per 100,000 [24]. Although this is no more applicable, it is still being utilized as the base of incidence estimates. This view has been questioned by a range of authors. Hans Rieder simply states in Epidemiologic Basis of Tuberculosis Control that "the determination of the risk of infection will not allow estimation of disease incidence in a community" [25]. In their 2008 report, the WHO also finally stopped using these kinds of calculations.

There are a number of reasons for this: At the most fundamental level, latent tuberculosis can be viewed as an equilibrium between host and bacillus [26]. In response to infection with $M$. tuberculosis, 
most persons mount a robust immune response that prevents active disease from occurring, and the bacterium avoids elimination. In most cases, the host response is sufficient to forestall active disease for a lifetime; however, occasionally the immune response fails and the infection reactivates to cause active disease. These complicated mechanisms cannot be calculated by a simple formula; the relationship between risk of infection and risk of reactivation and active disease is influenced by a range of factors of the bacilla, the host and the environment, and can be different from one population to another.

In conclusion; a model built on estimates derived from low-specific tuberculin surveys will probably not give us the true incidence. But what are the alternatives?

Among the models that have been developed to describe the infectious pool is the dynamic model of Chris Dye et al. (figure 1). Individuals enter into the pool when they develop active pulmonary $\mathrm{TB}$ and become infectious at a certain rate, and leave the pool as they start treatment, self-cure or die at certain rates. The size of the pool, and hence the burden of contagious cases, is defined by the relative size of these rates [27].

One achievable variable to estimate the infectious pool is treatment delay (TD), which is defined as the time from debut of the first symptom to the start of treatment after making a proper diagnosis. We conducted a literature review of TD in tuberculosis and found that TB patients are able to recall the onset of symptoms with acceptable accuracy [28].

\section{Materials and Methods}

In an attempt to operationalize the Dye model, we systematically recorded TD in a population of 18.1 million individuals in the Amhara Region of Ethiopia [29]. Three groups contribute to the infectious pool of TB: New TB cases, retreatment cases, and failures. Each patient's contribution to the infectious pool is equal to the number of days that he or she remains infectious; that is, the interval between the day the patient can recall the onset of symptoms and the day the patient starts treatment. Retreatment cases and failures remain infectious until they are cured or die. Our estimate of the infectious pool is based on a direct count of treatment delay days for each patient, and by estimating the number of undiagnosed cases, the total number of infectious days and hence an estimate of the infectious pool can be estimated.
A cross-sectional study was conducted between September 1, 2003, and December 31, 2003. The following zones were randomly selected from the eleven zones in Amhara, Ethiopia, as study sites: North and South Wollo, North Gonder, North Shewa, East Gojam and Bahir Dar. The study sites were all the five hospitals that are available in the selected zones. Besides this, we included 15 health care centers. We did not include any health stations because they are not diagnostic facilities for TB. Participants were consecutively included and interviewed immediately after diagnosis until the intended sample size was achieved. The data was collected by nurses and health officers who had been trained for the purpose. New sputum-smear positive (ss+) pulmonary TB patients aged 15 or more years were included in the original study on TD [30], but all categories of pulmonary TB patients were interviewed, and the data for the other patient categories were retrieved for the purpose of this study at a later stage. Sputum-smear negative (ss-) cases were diagnosed by a score system based on clinical evaluation aided by suggestive chest X-rays and/or lacking response to a course of broad-spectrum antibiotics. Cultivation of sputum is not available in the Amhara Region. Patient register card, TB registration books, and laboratory registers were cross-checked to assure the data quality and the collection of data was closely monitored by the study supervisors. The sample size was calculated using the formula required for determination of sample size for estimating single proportions. By taking a previous study performed in Ethiopia on TD, which showed a $58 \%$ proportion of more than one month delay, a $95 \%$ CI and a margin error of $5 \%$ of the sample size were calculated to 373 . In total 384 patients were included. A pretested structured questionnaire was used to collect information on sociodemographics, the major presenting symptoms of pulmonary $\mathrm{TB}$, the duration of the major presenting symptoms, and the date of the first health care visit. The major pulmonary symptoms included on the questionnaire were presence of cough for more than 3 weeks, production of sputum, chest pain, and hemoptysis. Patient register cards, TB registration books, laboratory registries and electronic data were crosschecked and validated to ensure the quality of data. The annual numbers for 2003 were interpolated. SPSS version 11.0 was used for analysis. 


\section{Results}

The results of our calculations for the year 2003 are presented in Table 1. The median infectious period for retreatment cases, defined as the time from cease of primary treatment to start of new treatment, was 75 days. Thus 49 retreatment cases contributed 3,675 days to the infectious pool. There were also 60 treatment failures, which because of their long infectious period of 365 days, made a substantial contribution of 21,900 days to the infectious pool. There were 5,013 new ss+ PTB cases, each with a median TD of 95 days. These cases made the largest contribution to the infectious pool (476,235 days). It was estimated that approximately $33 \%$ of all new ss+ PTB cases were undiagnosed or not notified by the NTP (see the discussion section for details on these calculations). The estimated infectious period of each untreated case was 60 days; thus these cases contributed 150,360 days to the infectious pool. The largest group of patients was the 6,159 ss- PTB cases who initiated TB treatment. Estimating their infectiousness as $20 \%$ of that of the ss+ cases (see the discussion section for details on these calculations), they had an infectious period of 20 days and contributed 123,180 days to the infectious pool. The total estimated infectious pool for the Amhara region in 2003 was 775,350 days, or 4,284/100,000 pop.

\section{Discussion}

There are two demanding variables in our calculations:

\section{The size and nature of the undiagnosed group}

The WHO calculated the CDR for new smear positive cases in Ethiopia to be $36 \%$ in 2003. As discussed in the background section, the CDR is built on old tuberculin surveys extrapolated to the present situation [1]. For several reasons it is unlikely that there are $64 \%$ undiagnosed cases. First, Pulmonary TB is a serious disease with characteristic symptoms. The patient seeks treatment, self-cures, or dies within a few months [31]. In many communities, the traditional belief as well as the experience of previous generations is that if you get TB you will die [32]. After the introduction of modern medicines these beliefs seem to be changing. Despite dire poverty and long distances to health care, most patients seek treatment, and they prefer allopathic (modern) medicine [33]. Our study on help-seeking behavior in Bangladesh found that more than $90 \%$ of patients with symptoms of TB preferred to visit an allopathic doctor first. Among the ten surveyed diseases, this was the highest preference score for modern medicine [32].

To estimate the size of the undiagnosed group a key question is "Are the non-detected mostly not ever detected, or are they not yet detected?" After Direct Observed Treatment Shortcourse (DOTS) is introduced in a country, the number of new cases identified proceeds through distinct transitional phases. This is illustrated by the development in the Sudan [33]. The national strategy was to first establish TB treatment facilities in the larger cities and main hospitals. This was followed by a steep rise in the number of diagnosed cases. After the introduction of the NTP at the district level, a further steady increase was expected, but did not happen. Instead, after an initial small increase in the case findings, mainly involving women and children, a plateau was reached. The symptoms of TB were serious enough that patients traveled a long distance for care. Thus the main proportion of patients were diagnosed at a time when treatment facilities were only available in the main cities [33], which indicates that most of the un-notified TB patients are not yet detected rather than not ever detected; they come forward and are notified sooner or later.

Ethiopia is an interesting case because TB drugs are not available outside the NTP. Private practitioners as well as traditional practitioners all refer their TB patients to the NTP for treatment [30]. This makes Ethiopian data on the undiagnosed group more reliable than data from most other high endemic countries where TB drugs are sold in the open market and a range of practitioners outside the NTP are providing treatment. A prevalence study was conducted by Shargie et al. in an area of Ethiopia with a well-organized TB control program that had functioned for more than ten years [34]. To the authors' surprise they found that only one-third of the total number of new ss++ were undiagnosed by the local TB control unit. In our study, we found the diagnostic delay of new ss+ TB cases to be 80 days [30]. Eleven weeks of infectiousness prior to diagnosis is five times as much as the two weeks of (gradually reduced) infectiousness after initiation of anti-TB treatment. Given that the non-detected patients are mostly not yet detected in the process of reaching a diagnosis, 33\% additional non-detected patients is in accordance with what we can expect, and we utilized this number as the base of our calculations of the size of the undiagnosed group. It must be emphasized that the relative number of un- 
notified cases, and the period they remain untreated, differs between countries and the regions within them, and must be carefully estimated before calculating the infectious pool. To estimate the number of undiagnosed $\mathrm{TB}$ cases and define the group that is not notified by the NTP but is still diagnosed and treated by private practitioners,

\section{The contribution of the sputum smear-negative} cases

Sputum smear-negative (ss-) PTB cases are generally less infectious, and their contribution to the infectious pool is not well studied. We based our calculations on two molecular studies: First a study in

Table 1. Annual infectious pool of TB cases in a population of 18.1 million in Amhara Region, Ethiopia.

\begin{tabular}{llll}
\hline Category & $\begin{array}{l}\text { Infectious period } \\
\text { (days)* }\end{array}$ & $\begin{array}{l}\text { Number of } \\
\text { patients }\end{array}$ & Number of infectious days \\
\hline Retreatment cases & 75 & 49 & 3675 \\
Failures & 365 & 60 & 21900 \\
Undiagnosed cases & $60 * *$ & $2506 * *$ & 150360 \\
New ss+ cases & 95 & 5,013 & 476235 \\
New ss- cases & $20 * * *$ & 6,159 & 123180 \\
\hline Total infectious pool & & 775350 \\
\hline Defined as the period from debut of symptoms to start of treatment \\
$* * *$ Estimated as 1:2 of all new ss+cases [34] \\
$* * *$ The infectiousness of ss- is estimated as $20 \%$ of the ss cases [35]; their infectious period is thus divided by five
\end{tabular}

hospitals and other health care facilities, the NTP needs to monitor their activities and have close cooperation.

In the validation phase of the new tool it is important to get an estimate of the undiagnosed cases, but one should not overemphasize this in the implementation; if repeated local prevalence studies are needed to validate this variable, the purpose of introducing a simple and feasible tool for a local TB program in a low-resource setting is not fulfilled. Why not then just perform prevalence studies? How can this new tool be utilized in areas where no proper or recent prevalence studies have been performed which constitute a majority of high-endemic areas? We believe that the most useful application of the new tool is to monitor trends, how the infectious pool changes, both to monitor the development of the local TB epidemic and to monitor the effectiveness of the local TB program. Hence, to monitor trends, the tool can be utilized even without knowing the percentage of undiagnosed cases. If a large majority of the undiagnosed cases are not yet detected rather than not ever detected, the significance of this group is not so much the number of cases but rather how long it takes before they are diagnosed, of which the tool gives a good estimate. The best way to effectively combat the epidemic is to focus on TD to reduce it.
Greater Vancouver, Canada, which determined that ss- cases are responsible for at least one-sixth of culture-positive episodes of TB transmission [35]. Secondly, a study in San Francisco, California, found that the source patient was ss- in at least $17 \%$ of the registered episodes of transmission, and it was estimated that ss- patients were $22 \%$ as likely as ss+ patients to transmit TB [36]. Studies such as these, which demand an almost complete overview of the transmission in a population and the utilization of advanced molecular techniques, have not been performed in any high-endemic country. Although we have no reason to believe that the infectiousness of ss- cases should be different from low- to highendemic areas, further hypothesis testing is needed. In our estimate, ss- PTB cases contribute to the infectious pool as $20 \%$ of the ss+ PTB cases, and in table 1 we have divided the ss- PTB cases' TD by 5 to estimate their contribution to the infectious pool.

Three recent studies estimated the median time to sputum culture conversion after first-line treatment to 28 days [37], 33 days [38] and 34 days [39], but the viability of the organisms rapidly deteriorate after treatment onset [40]. From several studies conducted in the 1960s, the praxis of releasing the hospitalized ss+ patient from isolation after two weeks on a regime including rifampicin was established, and is still the part of authoritative recommendations [41]. Taking into account the progressively reduced infectiousness, our estimates have added an 
infectious period of 15 days to each ss+ patient after the start of treatment.

As shown in our recently published review [28] of diagnostic and treatment delay in TB, our analysis revealed how complex it is to define the components of delay, and there were major differences between studies regarding definitions of onset of symptoms, first contact, and end of delay. A majority of the studies defined onset as the debut of any symptom; some studies defined onset as debut of cough; and one study defined onset as debut of any pulmonary symptom. A majority of the studies defined the first contact as the first visit to a qualified health care provider. Some studies defined the first contact as the time when the patient sought contact with any health care provider outside the household, including traditional practitioners. The studies also applied different definitions of the end of the delay. Some studies defined the end of health care system delay as the time when a correct diagnosis was made (diagnostic delay), while others defined it as the time the patient started treatment (treatment delay) or recorded both [28]. Realizing this variety of definitions, we saw the need to clearly define these variables in our study. Patient's delay (PD) was defined as the interval between the onset of any symptom and the time when the patient sought contact with any health care provider outside the household, including traditional practitioners. We chose to define end of delay as the day the patient started on treatment. Treatment delay (TD), which defines each patient's contribution to the infectious pool, was thus defined as the interval between debut of any symptom and the start of treatment. We justify this approach based on the fact that the infectious period is the period during which the patient coughs, and according to multiple studies, coughing is the cardinal first symptom of approximately $95 \%$ of ss+ patients $[30,42]$. Usually, patients can accurately define the onset of their symptoms, and even distinguish a new coughing pattern from previous coughing patterns with other causes such as Chronic Obstructive Lung Disease (COPD) or smoking [43]. The first symptom is important information and can be obtained with little extra effort by adding a question to patient registration forms. Moreover, this variable is not easily susceptible to "target perversion", which means that it is not so easy to manipulate to fulfill official demands and goals; it is information provided directly by the patients and recorded in the NTP files. The files can also easily be designed to record all five categories of patients that contribute to the infectious pool. Of the five patient categories, new ss+ TB cases contribute the majority of infectious days (77\%).

A more accurate understanding can be accomplished using TD as the main indicator. This is further illustrated by an Ethiopian intervention study wherein 32 local communities were randomly allocated to a control group of passive case findings or an intervention group of active case findings through monthly diagnostic outreach clinics, house visits, and public gatherings. In the intervention area, case finding was not significantly increased, but the TD was shortened. The proportion of patients with more than three months of symptoms was $41 \%$ in the intervention group and $63 \%$ in the control group. A sputum survey of 16,697 adults from the same area revealed a 2:1 ratio of ss+ cases receiving treatment to newly detected ss+ cases. The prevalence of ss+ TB was surprisingly low, only 78 per 100,000 [34, 44]. The studies were performed 10 years after the introduction of an effective DOTS program with $75 \%$ coverage and a $73 \%$ treatment success ratio [45].

One factor influencing the TD is the choice of health care practitioner. Patients who visit a hospital or a graduated private practitioner experience a shorter TD than do those who visit a village health worker, and patients who first visit a traditional healer experience the longest TD [42]. Longer TD is also associated with old age, rural residence, long distance to the clinic, low education level, and little knowledge of the symptoms of TB $[42,46,47]$.

\section{Conclusion}

There is currently no accurate methodology available to measure or monitor the infectious pool of $\mathrm{TB}$ in a population, both tuberculin and sputum surveys have substantial limitations and are resource demanding. We have proposed the utilization of a systematic recording of TD to achieve an estimate of the total number of infectious days as a possible alternative. This can be obtained by a careful recording of the date of debut of symptoms and the date of start of treatment for each patient, thus measuring his or her contribution to the infectious pool. We have demonstrated practically how such an estimate could be utilized by the local TB program of the Amhara region of Ethiopia as a quantifiable variable. When this is said, it must be admitted that there is still a substantial methodological uncertainty. Further validation studies are needed, especially regarding reproducibility of data, the patient's ability to recall the debut of symptoms, the size of the 
undiagnosed group, and the contribution of the sscases.

The nature of epidemics is that their reproductive rate has a decisive impact on the epidemiological trend. In the western world, small changes in the host-parasite balance such as improved housing caused the TB epidemic to decline long before specific treatment and preventive measures were available. At the same time, symptomatic sexually transmitted infections almost disappeared when effective antibiotics became available, with a sharp decline in the reproductive rate as the TD was reduced [48]. The same changes should occur with regard to $\mathrm{TB}$, and the crucial task is to reduce the unnecessary long treatment delay. In most cases the patients have been in touch with the formal health services as early as two weeks after the start of the symptoms. About $75 \%$ of the infectious pool could thus be attributed to delays in the health systems.

\section{Acknowledgements}

This study was supported by the Centre for Imported and Tropical Diseases, Ullevål University Hospital, and the Center for Prevention of Global Infections (Faculty of Medicine, University of Oslo, Norwegian Institute of Public Health, and Norwegian Health Services Research).

\section{References}

1. WHO. Global Tuberculosis Control: Surveillance, Planning, Financing: $\quad$ WHO 2008 . WHO/HTM/TB/2008.3932008.

2. Lalvani A, Nagvenkar P, Udwadia Z, Pathan AA, Wilkinson KA, Shastri JS, Ewer K, Hill AV, Mehta A, Rodrigues C. Enumeration of $\mathrm{T}$ cells specific for RD1-encoded antigens suggests a high prevalence of latent Mycobacterium tuberculosis infection in healthy urban Indians. J Infect Dis 2001;183:469-77.

3. Millennium Development Goals - A complete listing of the goals, targets, and indicators for MDGs 2003;Available rom: URL

4. Shigeto E, Tasaka H. [Tuberculin sensitivity to purified protein derivatives (PPD) from $\mathrm{M}$. intracellulare (PPD-B), M. kansasii (PPD-Y), M. fortuitum (PPD-Y) and M. tuberculosis (PPDs) among healthy volunteers]. Kekkaku 1993;68:283-91.

5. Menzies R, Vissandjee B, Amyot D. Factors associated with tuberculin reactivity among the foreign-born in Montreal. Am Rev Respir Dis 1992;146:752-6.

6. Rieder HL. Methodological issues in the estimation of the tuberculosis problem from tuberculin surveys. Tuber Lung Dis 1995;76:114-21.

7. Ewer K, Deeks J, Alvarez L, Bryant G, Waller S, Andersen P, Monk P, Lalvani A. Comparison of T-cell-based assay with tuberculin skin test for diagnosis of Mycobacterium tuberculosis infection in a school tuberculosis outbreak. Lancet 2003;361:1168-73.

8. Neuenschwander BE, Zwahlen M, Kim SJ, Engel RR, Rieder HL. Trends in the prevalence of infection with mycobacterium tuberculosis in Korea from 1965 to 1995: an analysis of seven surveys by mixture models. Int $\mathbf{J}$ Tuberc Lung Dis 2000;4:719-29.

9. Arnadottir T, Rieder HL, Trebucq A, Waaler HT. Guidelines for conducting tuberculin skin test surveys in high prevalence countries. Tuber Lung Dis 1996;77 Suppl 1:1-19.

10. Edwards LB, Acquaviva FA, Livesay VT, Cross FW, Palmer CE. An atlas of sensitivity to tuberculin, PPD-B, and histoplasmin in the United States. Am Rev Respir Dis 1969;99:Suppl:1-132.

11. WHO Tuberculosis Research Office. Further studies of geographic variation in naturally axquired tuberculin sensitivity. Bull World Health Organ 1955;22:63-83.

12. Britton WJ, Gilbert GL, Wheatley J, Leslie D, Rothel JS, Jones SL, Bradley P. Sensitivity of human gamma interferon assay and tuberculin skin testing for detecting infection with Mycobacterium tuberculosis in patients with culture positive tuberculosis. Tuberculosis (Edinb) 2005;85:137-45.

13. McConkey SJ, Youssef FG, Azem E, Frenck RW, Weil GJ. Evaluation of a rapid-format antibody test and the tuberculin skin test for diagnosis of tuberculosis in two contrasting endemic settings. Int J Tuberc Lung Dis 2002;6:246-52.

14. Pottumarthy S, Morris AJ, Harrison AC, Wells VC. Evaluation of the tuberculin gamma interferon assay: potential to replace the Mantoux skin test. J Clin Microbiol 1999;37:3229-32.

15. Pust RE, Erickson P. Determining Mycobacterium tuberculosis infection in high prevalence groups: a comparative study among Nigerian adults. Tubercle 1984;65:263-78.

16. Rangel-Frausto MS, Ponce-De-Leon-Rosales S, MartinezAbaroa C, Haslov K. Tuberculosis and tuberculin quality: best intentions, misleading results. Infect Control Hosp Epidemiol 2001;22:481-4.

17. Jentoft HF, Omenaas E, Eide GE, Gulsvik A. Comparing the adrenaline-Pirquet test with international PPD tuberculin tests. Respir Med 2001;95:205-11.

18. Cobelens FG, de Boer HE. [Specificity of the Mantouxreaction to the new tuberculin RT23 in relation to that of previously used PPD-RIVM; a comparative study in hospital staff]. Ned Tijdschr Geneeskd 1999;143:855-8.

19. Roth A, Sodemann M, Jensen H, Poulsen A, Gustafson P, Gomes J, Djana Q, Jakobsen M, Garly ML, Rodrigues A, Aaby P. Vaccination technique, PPD reaction and BCG scarring in a cohort of children born in Guinea-Bissau 20002002. Vaccine 2004;23:3991-8.

20. Dubuis M, Fiekert K, Johnston M, Neuenschwander BE, Rieder HL. A tuberculin skin test survey among Afghan children in Kabul. Int J Tuberc Lung Dis 2004;8:1065-72.

21. Cauthen GM, Pio A, ten Dam HG. Annual risk of tuberculous infection. 1988. Bull World Health Organ 2002;80:503-11; discussion 1-2.

22. Barr RG, Menzies R. The effect of war on tuberculosis. Results of a tuberculin survey among displaced persons in El Salvador and a review of the literature. Tuber Lung Dis 1994;75:251-9.

23. John TJ. Shortage of tuberculin in India: reason and remedy. Indian Pediatr 2004;41:293-4.

24. Styblo K. The relationship between the risk of tuberculous infection and the risk of developing infectious tuberculosis. Bulletin of the International Union against Tuberculosis and Lung Disease 1985;60:117-9.

25. Rieder H. Annual risk of infection with Mycobacterium tuberculosis. Eur Respir J 2005;25:181-5. 
26. Flynn JL. Immunology of tuberculosis and implications in vaccine development. Tuberculosis (Edinb) 2004;84:93-101.

27. Dye C, Garnett GP, Sleeman K, Williams BG. Prospects for worldwide tuberculosis control under the WHO DOTS strategy. Directly observed short-course therapy. Lancet 1998;352:1886-91.

28. Storla DG, Yimer S, Bjune GA. A systematic review of delay in the diagnosis and treatment of tuberculosis. BMC Public Health 2008;8:15.

29. Bahirdar ARSHB. Annual health servicereport. Bahirdar 2002.

30. Yimer S, Bjune G, Alene G. Diagnostic and treatment delay among pulmonary tuberculosis patients in Ethiopia: a cross sectional study. BMC Infect Dis 2005;5:112.

31. Styblo K. Epidemiology of tuberculosis. 2 ed. The Hague: Royal Netherlands Tuberculosis Association, 1991.

32. Storla DG, Bjune G, Hussain A. The SMNB Community Health Project - impact on the people's beliefs and practices regarding health and disease. Oslo: University of Oslo, 1996.

33. El-Sony AI, Mustafa SA, Khamis AH, Enarson DA, Baraka OZ, Bjune G. The effect of decentralisation on tuberculosis services in three states of Sudan. Int $\mathbf{J}$ Tuberc Lung Dis 2003;7:445-50.

34. Shargie EB, Yassin MA, Lindtjorn B. Prevalence of smearpositive pulmonary tuberculosis in a rural district of Ethiopia. Int J Tuberc Lung Dis 2006;10:87-92.

35. Hernandez-Garduno E, Cook V, Kunimoto D, Elwood RK, Black WA, FitzGerald JM. Transmission of tuberculosis from smear negative patients: a molecular epidemiology study. Thorax 2004;59:286-90.

36. Behr MA, Warren SA, Salamon H, Hopewell PC, Ponce de Leon A, Daley CL, Small PM. Transmission of Mycobacterium tuberculosis from patients smear-negative for acid-fast bacilli. Lancet 1999;353:444-9.

37. Dominguez-Castellano A, Muniain MA, Rodriguez-Bano J, Garcia M, Rios MJ, Galvez J, Perez-Cano R. Factors associated with time to sputum smear conversion in active pulmonary tuberculosis. Int J Tuberc Lung Dis 2003;7:4328.

38. Telzak EE, Fazal BA, Pollard CL, Turett GS, Justman JE, Blum S. Factors influencing time to sputum conversion among patients with smear-positive pulmonary tuberculosis. Clin Infect Dis 1997;25:666-70.

39. Fortun J, Martin-Davila P, Molina A, Navas E, Hermida JM, Cobo J, Gomez-Mampaso E, Moreno S. Sputum conversion among patients with pulmonary tuberculosis: are there implications for removal of respiratory isolation? J Antimicrob Chemother 2007;59:794-8.
40. Sirgel FA, Donald PR, Odhiambo J, Githui W, Umapathy KC, Paramasivan CN, Tam CM, Kam KM, Lam CW, Sole KM, Mitchison DA. A multicentre study of the early bactericidal activity of anti-tuberculosis drugs. J Antimicrob Chemother 2000;45:859-70.

41. Palomino JC, Leao SC, Ritacco V. Tuberculosis 2007 From basic science to patient care. Antwerpen: Institute of Tropical Medicine Antwerpen, 2007.

42. Lawn SD, Afful B, Acheampong JW. Pulmonary tuberculosis: diagnostic delay in Ghanaian adults. Int J Tuberc Lung Dis 1998;2:635-40.

43. Storla DG, Rahim Z, Islam MA, Plettner S, Begum V, Mannsaaker T, Myrvang B, Bjune G, Dahle UR. Heterogeneity of Mycobacterium tuberculosis isolates in Sunamganj District, Bangladesh. Scand $\mathbf{J}$ Infect Dis 2006;38:593-6.

44. Shargie EB, Lindtjorn B. DOTS improves treatment outcomes and service coverage for tuberculosis in South Ethiopia: a retrospective trend analysis. BMC Public Health 2005;5:62.

45. Shargie EB, Morkve O, Lindtjorn B. Tuberculosis casefinding through a village outreach programme in a rural setting in southern Ethiopia: community randomized trial. Bull World Health Organ 2006;84:112-9.

46. Lin HP, Deng CY, Chou P. Diagnosis and treatment delay among pulmonary tuberculosis patients identified using the Taiwan reporting enquiry system, 2002-2006. BMC Public Health 2009;9:55.

47. Huong NT, Vree M, Duong BD, Khanh VT, Loan VT, Co NV, Borgdorff MW, Cobelens FG. Delays in the diagnosis and treatment of tuberculosis patients in Vietnam: a crosssectional study. BMC Public Health 2007;7:110.

48. Lewis M. Thorns on the rose: the history of sexually transmitted diseases in Australia in international perspective. Canberra: Australian Govt. Publ. Service, 1998.

\section{Corresponding Author}

Dag Gundersen Storla

Department of International Health

Institute of General Practice and Community Medicine

University of Oslo

PO Box 1130 Blindern

N-0318 Oslo

Norway

Tel: +4790215049

E-mail: dgstorla@online.no

Conflict of interests: No conflict of interest is declared. 